\title{
Instability and transport of metal catalyst in the growth of tapered silicon nanowires
}

\author{
Linyou $\mathrm{Cao}^{1}$, Bora Garipcan ${ }^{2}$, Jennifer S. Atchison ${ }^{1}$, Chaoying Ni, ${ }^{3}$ Bahram Nabet $^{1,4}$, Jonathan E. Spanier ${ }^{1,4, *}$ \\ ${ }^{1}$ Department of Materials Science $\&$ Engineering,Drexel University, Philadelphia PA 19104; \\ ${ }^{2}$ Department of of Chemical Engineering, Bioengineering Divison, \\ Hacettepe University, Beytepe, Ankara, 06800 TURKEY; \\ ${ }^{3}$ Department of Materials Science and Engineering, University of Delaware, Newark DE 19716; \\ ${ }^{4}$ Department of Electrical and Computer Engineering, Drexel University, Philadelphia PA 19104 \\ *to whom correspondence should be addressed: spanier@drexel.edu
}

\section{GAS FLOW IN FURNACE TUBE}

The flow field in a quartz chemical vapor deposition (CVD) furnace tube is subject to the fluid dynamics associated with the tube geometry, pressure, temperature, and flow rate as well as thermodynamic properties of the gas components[1]. The mean velocity of the flow $\overline{v_{g}}$ depends on the pumping rate, the tube geometry, pressure and flow rate of the gas[2],

$$
S=\frac{T_{\text {out }}}{T_{\text {in }}} \cdot \frac{P_{\text {in }}}{P_{\text {out }}} \cdot \frac{d V}{d t}=\overline{v_{g}} \cdot A
$$

where $S$ is the pumping speed, $d V / d t$ is gas flow rate, $T_{\text {in }}, P_{\text {in }}$ and $T_{\text {out }}, P_{\text {out }}$ are temperature and pressures of the gas at the input entrance and the output orifice, respectively, and $\mathrm{A}$ is the cross-sectional area of the tube. Substituting typical values $\left(P_{\text {in }}=40 \mathrm{psi}, \mathrm{P}_{\text {out }}=1\right.$ torr, $\mathrm{T}_{\text {in }}=293 \mathrm{~K}$ and $\mathrm{T}_{\text {out }}=923 \mathrm{~K}$ ), one obtains $\overline{v_{g}}=20.9$ $\mathrm{m} / \mathrm{s}$. In addition, the mean free path of gas molecules $\lambda$ can be obtained using[2]

$$
\lambda=\frac{R T}{\sqrt{2} \pi d^{2} N_{A} P}
$$

where $d$ is the diameter of the gas molecule, $P$ is pressure of the gas flow and $N_{A}, R$, and $T$ have their conventional meanings. Using $(\mathrm{S} 2)$ and typical values, $\lambda$ is calculated to be on the order of $\lesssim 50 \mu \mathrm{m}$, which is much smaller than the inner diameter of the tube $b(=$ $2.54 \mathrm{~cm}$ ), indicating that the gas flow is viscous flow [3]. The dimensionless Reynolds number can be used to identify the regime of viscous flow further[3]:

$$
R e=\sum_{i}^{n}\left(\frac{\rho_{g}}{\mu_{g}}\right)_{i} \overline{v_{g}} \cdot b=\sum_{i}^{n}\left(\frac{P_{g} M_{g}}{\mu_{g}}\right)_{i} \frac{\overline{v_{g}} \cdot b}{R T}
$$

where $\left(\rho_{g}\right)_{i},\left(\mu_{g}\right)_{i},\left(P_{g}\right)_{i}$, and $\left(M_{g}\right)_{i}$ are mass density, viscosity, partial pressure and molar mass of the $i^{\text {th }}$ component in the gas mixture. In our experiments, the gas mixture mainly includes carrier gases (e.g. $\mathrm{N}_{2}$ ), and process gases (95\% vol. He plus $\left.5 \% \mathrm{SiH}_{4}\right) ; P_{g}$ for other components not purposely introduced (such as $\mathrm{O}_{2}$ and $\mathrm{Au}$ vapor) are so small that we have neglected their contribution to the estimate of $R e$. The value obtained for $R e(=19.3)$ indicates our conditions correspond to laminar flow [4].

The velocity profile in laminar flow possesses a parabolic shape across the cross-section of the tube, and near the substrate the velocity of gas is nearly zero. The thickness of the stagnant layer $\delta$ in which the flow velocity changes from that of the bulk gas to zero increases along downstream direction is approximately[1] $\delta \approx(x b / R e)^{1 / 2}$ where $x$ is the distance from the edge of the boat closest to the inlet and along the flow direction. However, we assume here for simplicity that the thickness of the stagnant layer is zero and the velocity is uniform at the cross section. Taking into account of blocking effect of the boat, we may estimate the mean velocity of gas flow passing by the boat is $\bar{v} \approx 1.5 \overline{v_{g}}=30.4 \mathrm{~m} / \mathrm{s}$.

\section{CALCULATION OF EVAPORATION RATE FROM AN AU NANOPARTICLE}

The evaporation of a liquid droplet in a gas flow can be expressed as ${ }^{5}$

$$
\frac{d D^{2}}{d t}=\left(\frac{d D^{2}}{d t}\right)_{0}\left[1+0.276\left(N_{\mathrm{Re}}\right)^{1 / 2}\left(N_{S h}\right)^{1 / 3}\right]
$$

where $\left(d D^{2} / d t\right)_{0}$ represents the evaporation rate in still air, and $N_{\text {Re }}$ and $N_{S h}$ are the dimensionless Reynolds and Sherwood numbers for gold vapor ${ }^{5}$

$$
N_{\operatorname{Re}}=\frac{\rho_{A u(g)} v_{g} b}{\mu_{A u(g)}}
$$

$$
N_{S h}=\sqrt{\frac{2}{\pi}}\left(\frac{\rho_{A u(g)}(b / 2)^{3}}{9 \mu_{A u(g)} D_{d i f f}^{A u(g)}}\right)^{1 / 2}
$$


Here $\rho_{A u(g)}, \mu_{A u(g)}$ and $D_{d i f f}^{A u(g)}$ are, respectively, the density, viscosity and diffusion coefficient of gold vapor, the magnitudes of which are on the order of $10^{-14} \mathrm{~kg} / \mathrm{m}^{3}$, $10^{-5} \mathrm{~N} \cdot \mathrm{s} / \mathrm{m}^{2}$, and $10^{-5} \mathrm{~m}^{2} / \mathrm{s}$, respectively. These yield values for $N_{\operatorname{Re}}$ and $N_{S h}$ which are negligibly small such that $(\mathrm{S} 4)$ can be rewritten $d D^{2} / d t \approx\left(d D^{2} / d t\right)_{0}$. Assuming saturated vapor pressure and no temperature gradient at the surface of the catalyst droplet, the evaporation rate in still air can be written[5]

$$
\left(\frac{d D^{2}}{d t}\right)_{0}=2 \frac{\Gamma_{A u(g)}}{\rho_{A u(l)}} \ln \left(1+\frac{m_{A u(g), 0}-m_{A u(g), \infty}}{1-m_{A u(g), 0}}\right)
$$

where $\Gamma_{A u(g)}$ is exchange coefficient of gold vapor in the gas flow, which is equal to the product of the diffusion coefficient $D_{\text {diff }}^{A u(g)}$ and the mass density of gold vapor $\rho_{A u(g)}$. The exchange coefficient $\Gamma_{A u(g)}=D_{d i f f}^{A u(g)}$. $\rho_{A u(g)} \approx 10^{-19} \mathrm{~kg} \cdot \mathrm{m}^{-1} \cdot \mathrm{s}^{-1}, m_{A u(g)}$ is the mass fraction of gold vapor in the gas flow, and the subscripts 0 and $\infty$ denote the value of $\mathrm{m}_{A u(g)}$ at the droplet surface and far away from the droplet, respectively. Taking $m_{A u(g), \infty}=0$, Eq. (S7) can be written

$$
\left(\frac{d D^{2}}{d t}\right)_{0}=-2 \frac{\Gamma_{A u(g)}}{\rho_{A u(l)}} \ln \left(1-m_{A u(g), 0}\right)
$$

Assuming the gas mixture is as an ideal gas solution, one obtains for $m_{A u(g)}$ according to Raoult's Law,

$$
m_{A u(g), 0}=\frac{\chi_{A u(g)} M_{A u(g)}}{\sum_{i=1}^{n} \chi_{i} M_{i}}
$$

where $\chi_{i}, M_{i}$ are molar fraction and molar mass of $\mathrm{i}^{\text {th }}$ component in the gas flow, and the former is given by

$$
\chi_{A u(g)}=\frac{P_{A u(g)}(T)}{P}
$$

Where $P$ is total pressure of the gas flow, and $P_{A u(g)}(T)$ is the vapor pressure of gold at temperature $T$. Using the Clausius-Clayperon equation and a typical growth temperature $(\sim 923 \mathrm{~K}) P_{A u(g)}(T) \approx 3.21 \times 10^{-10} \mathrm{~Pa}$, yielding a value using Eq. (S9) of $m_{A u(g), 0} \approx 7.9 \times 10^{-10}$. Since $\ln (1-x) \approx-x$ when $x<<1$, Eq. (S8) can be rewritten

$$
\left(\frac{d D^{2}}{d t}\right)_{0}=2 \frac{\Gamma_{A u(g)} m_{A u(g), 0}}{\rho_{A u(l)}}
$$

With the above and $\rho_{A u(l)}=1.932 \times 10^{4} \mathrm{~kg} \cdot \mathrm{m}^{-3}$, one obtains an estimate for the evaporation rate of $\left(d D^{2} / d t\right)_{0}$ $\approx 10^{-32} \mathrm{~m}^{2} / \mathrm{s}$, which corresponds to a rate of change in thickness at the surface of the droplet of $\sim 10^{-14} \mathrm{~nm} / \mathrm{s}$. Therefore the, contribution of evaporation (and condensation) to the observed transport of $\mathrm{Au}$ atoms to other substrates is negligibly small.

\section{POSSIBLE ROLE OF HYDRODYNAMIC FORCE}

To consider the possible role of the hydrodynamic force in the dissociation of the droplets, we model the twophase flow in our experiments as a droplet in radius of $\mathrm{r}$ located in an unbounded fluid with flow speed $\bar{v}$, viscosity $\mu_{g}$ and the interfacial tension acting between the two fluid phases $\gamma$. As per the Rayleigh limit, the stability of a droplet here is determined by balancing between three forces: the drag force (or inertia force), the viscous force and the surface tension force[6]. The drag force and viscous force tends to deform the catalyst droplet, and to finally detach it or cause it to dissociate, while surface tension force opposes this kind of deformation, keeping the droplet in its original shape. Breakup of the droplet will occur if the holding force of surface tension is exceed by the sum of the deforming force[6], or $F_{D}+F_{V}>F_{S}$. Because of the small size of the droplet, the fluid motion with respect to the droplet is governed by Stokes law, so that $F_{D}=\sum_{i}^{n} 6 \pi r\left(\mu_{g}\right)_{i} \bar{v}[7]$.

The viscous and surface tension forces can be written[7] $F_{V}=\sum_{i}^{n} 2 \pi r\left(\mu_{g}\right)_{i} \bar{v}$, and $F_{S}=2 \pi r \gamma$, and so $F_{V}+F_{D}=$ $\sum_{i}^{n} 8 \pi r\left(\mu_{g}\right)_{i} \bar{v}$. Substituting typical values $(\gamma=0.85$ $\mathrm{N} / \mathrm{m}, \mu_{g}=0.038 \mathrm{cP}$, and $\left.\bar{v}=30.4 \mathrm{~m} / \mathrm{s}\right)$, one obtains $2 \pi r \gamma \approx 1.7 \pi r$ and $\sum_{i}^{n} 8 \pi\left(\mu_{g}\right)_{i} \bar{v} r=9.3 \times 10^{-2} r$. Thus $F_{D}+F_{V}<<F_{S}$, and therefore the drag force imposed by the gas flow cannot be the origin of the observed droplet breakup.

[1] Pierson, H.O. Handbook of Chemical Vapor DepositionPrinciples, Technology and Application; William Andrew Publishing: Norwich, 1999.

[2] Mahan, J. E. Physical Vapor Deposition of Thin Films; John Wiley \& Sons: New York, 2000.

[3] Batchelor, G.K. An Introduction to Fluid Dynamics; Cambridge Univ. Press: Cambridge, 1967.

[4] Streeter, V.L. Handbook of Fluid Dynamics; McGrawHill: New York, 1961.

[5] Crowe,C.; Sommerfeld, M.; Tsuji, Y. Multiphase Flows with Droplets and Particles; CRC: Boca Raton, 1998. 
[6] Stone, H.A. Annu. Rev. Fluid Mech. 1994, 26, 65.

[7] Guyon, E.; Hulin, J.; Petit, L.; Mitescu, C.D. Physical Hydrodynamics; Oxford Univ. Press: Oxford, 2001.

FIG. 1: Representative secondary emission scanning electron microscopy (SEM) images of samples illustrating suppression of Au catalyst transfer and growth of SiNCs on initially catalyst-free adjacent substrates. (a), Representative SEM images of SiNCs grown with $\mathrm{PH}_{3}$ as a dopant gas in addition to $\mathrm{SiH}_{4}$ as described in the main text. (b), SEM image of a representative morphology collected from an initially catalyst-free substrate placed next to and downstream in the growth furnace as described in the main text. (c), SEM image of a representative morphology collected under identical growth conditions, but from a bare, catalyst-free substrate in the absence of a substrate possessing Au catalyst nanoparticles. The morphology seen in $(b)$ is consistent with that observed in $(c)$, and is due to homogeneous decomposition of $\mathrm{SiH}_{4}$ and the resulting island growth. The sample imaged in $(b)$ and other similar samples were mapped at sufficient magnification to detect SiNCs; the appearance of SiNCs in (a) coupled with their total absence on sample $(b)$, and the similarity in appearance of $(b)$ to $(c)$ supports our model of droplet break-up due to a build-up of electrostatic charge as described in the main text.
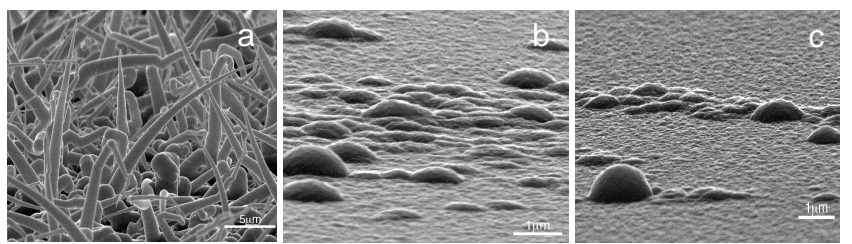